\title{
MODELOS DE PREVISÃO PARA TEMPERATURA E SALINIDADE NO FENÔMENO DE RESSURGÊNCIA: ANÁLISE DOS DADOS DA BOIA 1900’S3400’W NO PERÍODO ENTRE 2005 E 2014
}

\author{
Paulo Henrique Couto Simões \\ Universidade do Estado do Rio de Janeiro - UERJ \\ Rua São Francisco Xavier 524, $6^{\circ}$ andar, sala 6028-B, Maracanã, RJ, CEP: 20550-900 \\ ph.simoes@gmail.com \\ José Fabiano da Serra Costa \\ Universidade do Estado do Rio de Janeiro - UERJ \\ Rua São Francisco Xavier 524, $6^{\circ}$ andar, sala 6028-B, Maracanã, RJ, CEP: 20550-900 \\ fabiano@ime.uerj.br \\ Marcello Montillo Provenza \\ Universidade do Estado do Rio de Janeiro - UERJ \\ Rua São Francisco Xavier 524, $6^{\circ}$ andar, sala 6028-B, Maracanã, RJ, CEP: 20550-900 \\ mprovenza@gmail.com \\ Vinicius Layter Xavier \\ Universidade do Estado do Rio de Janeiro - UERJ \\ Rua São Francisco Xavier 524, $6^{\circ}$ andar, sala 6028-B, Maracanã, RJ, CEP: 20550-900 \\ viniciuslx@ime.uerj.br \\ Jorge Luiz de Jesus Goulart \\ Universidade do Estado do Rio de Janeiro - UERJ \\ Rua São Francisco Xavier 524, 6º andar, sala 6028-B, Maracanã, RJ, CEP: 20550-900 \\ jorge_goulart@outlook.com
}

\section{RESUMO}

Os mais de sete mil quilômetros do litoral brasileiro constituem uma zona de riqueza ímpar do nosso país onde se concentra grande parte da população brasileira. É na região costeira onde se realiza grande parte da pesca, da navegação, da extração de petróleo, do lazer e do turismo do Brasil. Nesse cenário, um conhecimento das condições oceânicas existentes ao longo do nosso litoral torna-se cada vez mais necessário. Diversos programas incentivam e executam atividades relacionadas ao estudo dos oceanos. Nesse trabalho, será analisada a base de dados do GOOS-BRASIL que é um sistema nacional de observação dos oceanos. O objetivo do presente estudo é estudar a série temporal entre 2005 e 2014 das temperaturas e salinidades médias a um metro de profundidade, coletadas da boia ATLAS (posição geográfica $19^{\circ} 00 \mathrm{~S} 34^{\circ} 00 \mathrm{~W}$ ) da rede PIRATA. Inicialmente foram feitas as análises de estacionariedade e sazonalidade, para posteriormente elaborar as previsões. Foram utilizados os modelos de alisamento exponencial e avaliados pelas métricas do Erro Quadrático Médio e da Média Absoluta Percentual de Erro. 
Palavra-chave: Previsão; Séries Temporais; Modelos de Alisamento; Temperatura; Salinidade.

\begin{abstract}
The more than seven thousand kilometers of the Brazilian coast constitute a unique wealth zone of our country where a large part of the Brazilian population is concentrated. It is in the coastal region where much of Brazil's fishing, navigation, oil extraction, leisure and tourism is carried out. In this scenario, a knowledge of the ocean conditions existing along our coast becomes increasingly necessary. Several programs encourage and carry out activities related to the study of the oceans. In this work, the GOOS-BRAZIL database which is a national ocean observation system will be analyzed. The objective of the present study is to study the time series between 2005 and 2014 of average temperatures and salinities at one meter depth, collected from the ATLAS buoy (geographical position $19^{\circ} 00 \mathrm{~S} 34^{\circ} 00 \mathrm{~W}$ ) of the PIRATA network. Initially the stationarity and seasonality analyzes were made, to later elaborate the predictions. Exponential smoothing models were used and evaluated by the Mean Square Error and Absolute Mean Error Percentage metrics.
\end{abstract}

Keywords: Prediction; Times Series; Smoothing Models; Temperature; Salinity.

\title{
Como Citar:
}

SIMÕES, Paulo Henrique Couto; SERRA COSTA, José Fabiano da; PROVENZA, Marcello Montillo; XAVIER, Vinicius Layter; GOULART, Jorge Luiz de Jesus. Modelos de previsão para temperatura e salinidade no fenômeno de ressurgência: análise dos dados da boia 1900'S34ㅇ' $\mathrm{W}$ no período entre 2005 e 2014. In: SIMPÓSIO DE PESQUISA OPERACIONAL E LOGÍSTICA DA MARINHA, 19., 2019, Rio de Janeiro, RJ. Anais [...]. Rio de Janeiro: Centro de Análises de Sistemas Navais, 2019.

\section{INTRODUÇÃO}

O Brasil possui um litoral com mais de 7.400 km de extensão. Ao longo desta costa são desenvolvidas diversas atividades, muitas das quais de relevância socioeconômicas, tais como: atividades portuárias, de pesca e aquicultura, bem como a exploração de recursos minerais. Muitas das principais cidades brasileiras são litorâneas, têm no turismo e nas atividades recreativas importantes fontes de renda e são vulneráveis às variações do nível do mar para fins de saneamento e de defesa civil e demarcação do Patrimônio da União.

Dentro deste contexto, faz-se importante o monitoramento das variações climáticas do mar. Durante dez anos, entre 1987 e 1996, a Diretoria de Hidrografia e Navegação iniciou o projeto de Levantamento da Plataforma Continental (LEPLAC), em parceria com a PETROBRÁS e universidades brasileiras. Os dados oceanográficos coletados, ao longo de toda a margem continental brasileira, subsidiaram a confecção de mapas para o estabelecimento do limite da Plataforma Continental. É importante a nação brasileira conhecer melhor os detalhes do que representa nossa área marítima para seus interesses. A Amazônia Azul, que nos rodeia com cerca de $8.500 \mathrm{~km}$ de litoral e se estende mar adentro por distâncias que, às vezes, ultrapassam as 200 milhas marítimas.

Conforme estabelecido na Convenção das Nações Unidas sobre o Direito do Mar (ratificada por quase 100 países - inclusive o Brasil) todos os bens econômicos existentes no seio da massa líquida, sobre o leito do mar e no subsolo marinho, ao longo de uma faixa 
litorânea de 200 milhas marítimas de largura, na chamada Zona Econômica Exclusiva (ZEE), constituem propriedade exclusiva do país ribeirinho. Em alguns casos, a Plataforma Continental (PC) - prolongamento natural da massa terrestre de um Estado costeiro ultrapassa essa distância, podendo estender a propriedade econômica do Estado a até 350 milhas marítimas. Essas áreas somadas (a ZEE mais a PC) caracterizam a imensa Amazônia Azul, medindo quase 4,5 milhões de $\mathrm{km}^{2}$, o que acrescenta ao país uma área equivalente a mais de $50 \%$ de sua extensão territorial.

Diversos programas, em nível mundial, incentivam e executam atividades relacionadas ao estudo dos oceanos. Um destes programas é o Global Sea Level Observing System (GLOSS - Sistema Global de Observação do Nível do Mar), coordenado pela organização World Meteorological Organization (WMO - Organização Meteorológica Mundial) e pela Intergovernmental Oceanographic Commission (IOC - Comissão Oceanográfica Intergovernamental). O IOC tem como objetivo primordial o estabelecimento de um sistema estratégico permanente para o fornecimento de informação de alta qualidade sobre o nível dos mares e o monitoramento de suas mudanças globais.

O equipamento básico de toda estação que monitora o nível do mar é o Marégrafo, que essencialmente consiste em um medidor que detecta e registra essa variável através de diversos sistemas, tais como: flutuadores, sensores de pressão, contatos elétricos, pulsos acústicos, bolhas, radar etc. É extremamente importante que as estações realizem não só medições redundantes de nível do mar, como também medições de outros parâmetros meteorológicos e oceanográficos (vento de superfície, temperatura da superfície, condutividade de superfície (salinidade), temperatura do ar, umidade relativa do ar, radiação de ondas curtas, precipitação, temperatura de sub-superfície (10m profundidades até $500 \mathrm{~m})$, condutividade de sub-superfície (3m profundidades até $500 \mathrm{~m}$ ), e pressão a 300 e $500 \mathrm{~m}$ ).

Um requisito básico do GLOSS é que toda estação deve realizar e registrar medições com uma acurácia melhor do que um centímetro sob todas as condições de maré, correntes de maré e todas as condições ambientais que possam ocorrer (chuva, temporais, ressacas e outras). O controle geodésico de cada estação deverá incluir a realização periódica de nivelamento geométrico de alta precisão, em intervalos de seis meses a um ano. As medições devem ser feitas seguindo as normas e especificações vigentes no Brasil (DecretoLei $\mathrm{n}^{\circ} 243$, 1967). A boia localizada na posição geográfica $19^{\circ} 00^{\prime} S 34^{\circ} 00^{\prime} \mathrm{W}$ (Figura 1) que será objeto desse estudo pertence à rede Pilot Research Moored Array in the Tropical Atlantic (PIRATA - Pesquisa Piloto Ancorada no Atlântico Tropical), que é uma sub-rede do sistema GLOSS. As boias utilizadas são do tipo Autonomous Temperature Line Acquisition System (ATLAS - Sistema de Aquisição de Linha de Temperatura Autônoma).

Figura 1: Posição geográfica da boia utilizada no presente estudo. 


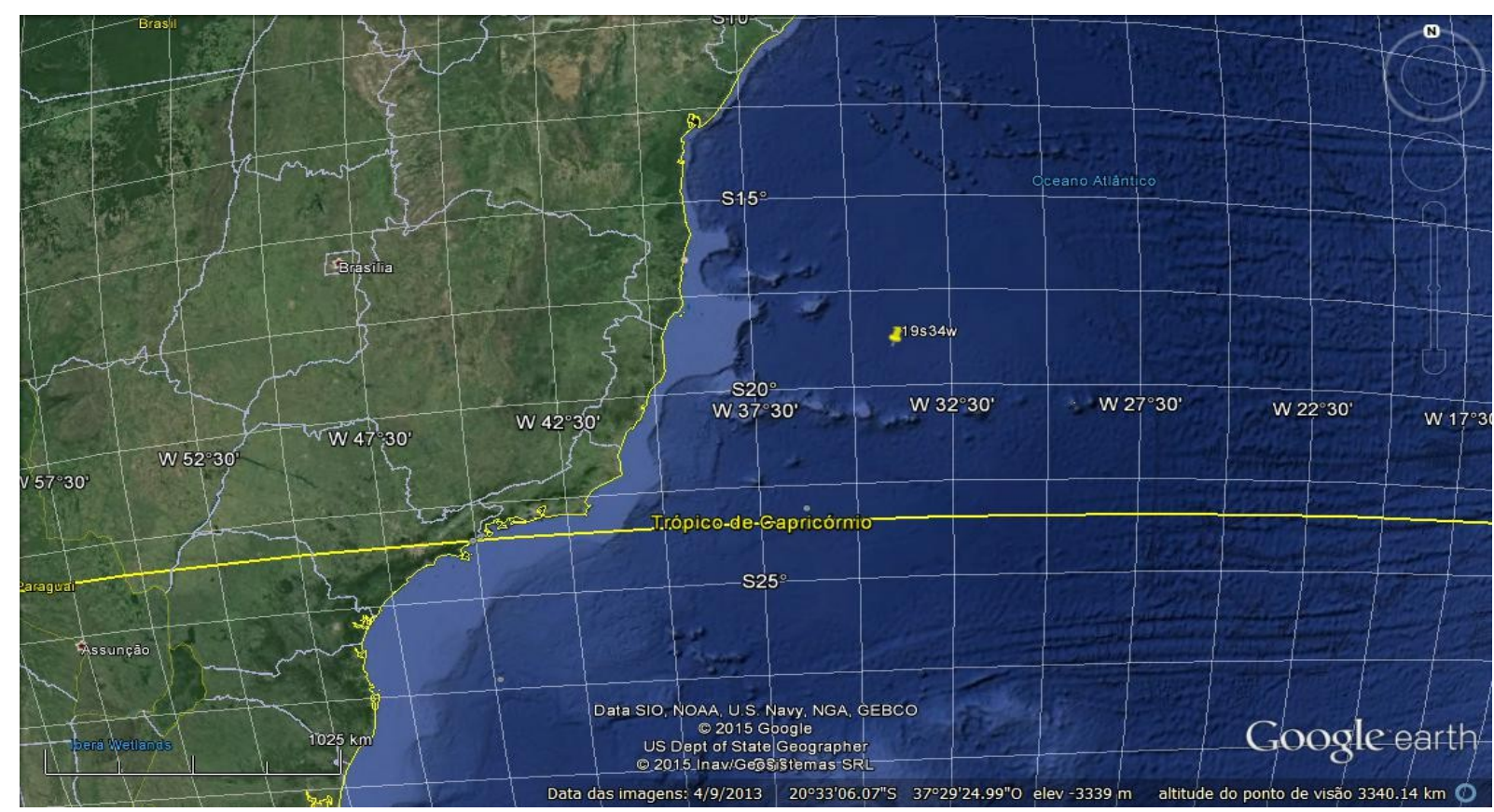

Fonte: Google Earth, 2015.

\section{OBJETIVO}

O presente estudo tem como principal finalidade testar dois tipos de abordagem estatística para análise e previsão de séries temporais em quatro modelos de alisamento exponencial e identificar o que melhor se ajusta aos dados da série composta pela temperatura média mensal e pela salinidade média mensal na posição geográfica $19^{\circ} 00^{\prime} \mathrm{S} 34^{\circ} 00^{\prime} \mathrm{W}$ no período entre 2005 e 2014. Para a escolha do melhor modelo são utilizadas medidas estatísticas de análise de desempenho aplicadas as previsões obtidas para o período entre 2013 e 2014.

\section{PROPRIEDADES OCEANOGRÁFICAS DO ESTUDO}

O principal fenômeno observado nesse estudo será o da ressurgência que consiste no afloramento de águas profundas com temperaturas frias, que por estarem em uma região onde a luz solar não alcança (afótica) são ricas em nutrientes e sais minerais oriundos da deposição, no fundo do mar, dos dejetos dos animais, seus corpos mortos, carapaças, etc., e pela ação das bactérias anaeróbicas tudo é remineralizado, sendo transformado nos chamados sais nutrientes, que através do fenômeno da ressurgência subirão à superfície reiniciando o ciclo.

Defant (1961) ao apresentar as distribuições de temperatura, de salinidade e densidade dos oceanos, menciona o Atlas de Bohnecke como a representação mais compreensiva sobre as variações dessas propriedades na superfície do oceano atlântico. Na construção desse atlas, Bohnecke utilizou-se das informações obtidas durante a expedição de 1925 a 1927 do navio alemão Meteor, e também de todas as informações disponíveis de temperatura e de salinidade observadas no Oceano Atlântico.

O trabalho de Mascarenhas Jr., Miranda e Rock (1971) constatou a ocorrência do fenômeno de ressurgência em Cabo Frio, através de um forte gradiente horizontal de temperatura na superfície do mar, e que, posteriormente a Marinha Brasileira em seu programa IGY - 1957 (International Geophysical Year) observou o mesmo fenômeno ao sul de Cabo Frio e também na costa do Espírito Santo.

Emilsson (1961), baseado nos dados de três cruzeiros oceanográficos realizados em 1956, sugeriu que a ressurgência se mantém pelo processo termo-halino causado pela 
radiação solar que, aquecendo a água ressurgente (fria e de baixa salinidade), provoca a diminuição de densidade. Ora, para que a estabilidade dinâmica seja mantida, as águas aquecidas são transportadas ao largo, ocasionando movimentos ascendentes de águas de camadas mais profundas que, por sua vez, sofrem diminuição de densidade. Este processo deve ocorrer com continuidade para ocasionar anomalias na distribuição das propriedades citadas. Tal mecanismo deve ser, a par com ventos dominantes, o fator predominante de uma ressurgência permanente e periódica nas vizinhanças de outras zonas tropicais e subtropicais dos oceanos.

\subsection{Sistema ATLAS}

Segundo De Andrade e Costa (2011), atualmente no Brasil pode ser constatada uma crescente necessidade de obtenção de dados meteoceanográficos. Neste contexto, observa-se que essa demanda é mais expressiva nos setores de Óleo e Gás, Offshore, Naval, Portos e Estaleiros, Mineração, Siderurgia, Usinas Hidrelétricas, Termelétricas e Nucleares; assim como, Institutos de Pesquisas Científico-Acadêmicos. A obtenção de dados meteoceanográficos tem como objetivo aumentar a compreensão dos fenômenos e condições meteorológicas e oceanográficas, sendo que, tais dados são coletados principalmente em regiões onde possíveis projetos dos setores supracitados serão desenvolvidos, como por exemplo, plataformas de produção de petróleo, estaleiros, portos, etc.

Além disso, com este conhecimento também é possível prevenir possíveis avarias a estruturas que venham ser instaladas em locais de interesse tanto em regiões costeiras ou offshore. Ao mesmo passo da necessidade de obtenção de parâmetros meteoceanográficos, ocorre a necessidade de desenvolvimento de equipamentos nacionais para coleta de tais dados, já que, a maioria dos equipamentos disponíveis no mercado é de origem estrangeira, o que acarreta no aumento do custo e fragilidade do projeto ou, em alguns casos, pode até inviabilizar sua execução. A Figura 2 mostra um exemplo de boia ATLAS.

Figura 2: Boia ATLAS usada no projeto PIRATA fabricada pelo PMEL/NOAA.

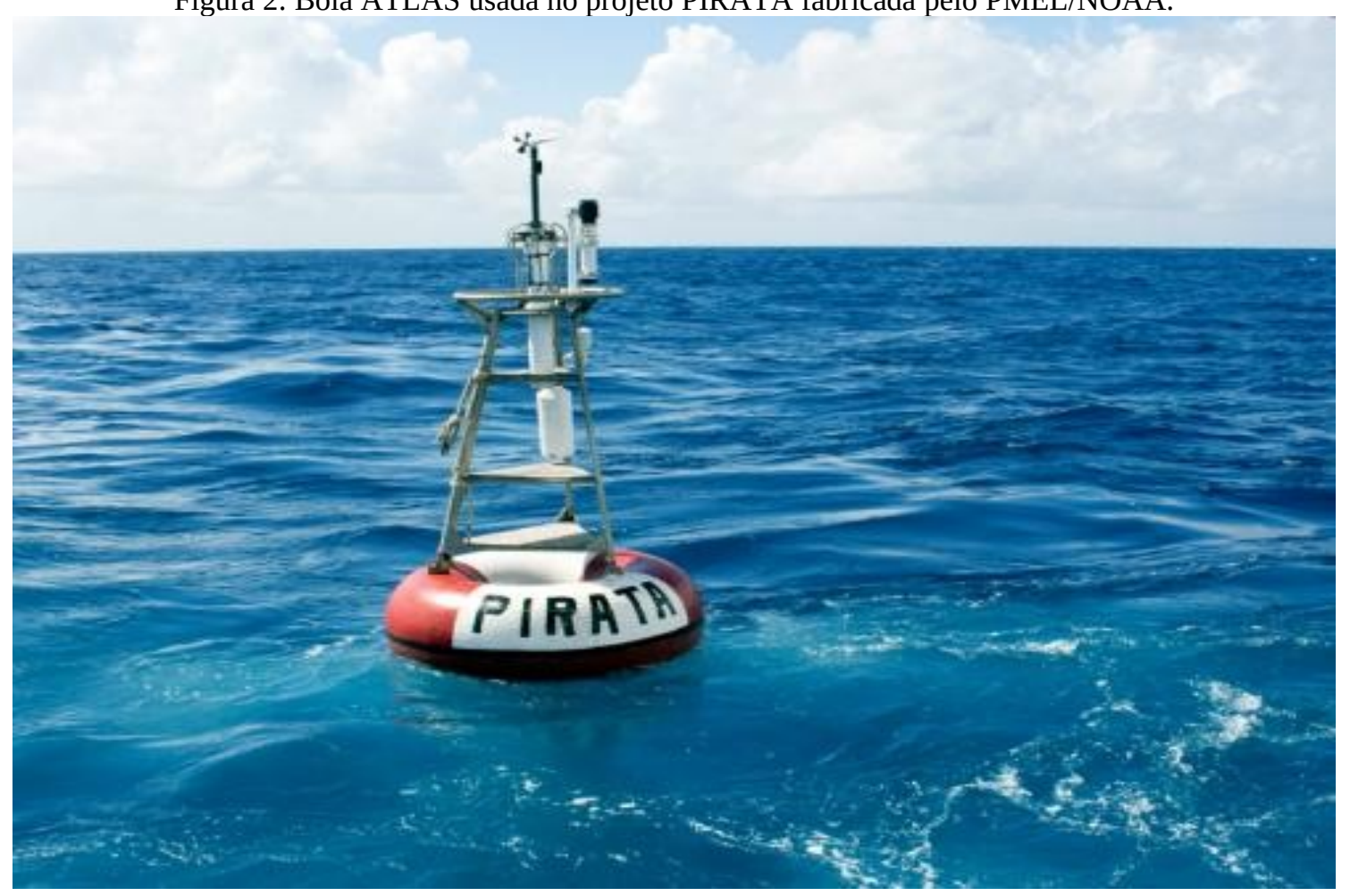

Fonte: Marinha do Brasil, 2019. 
Baseado nos moldes do Projeto PIRATA, o Instituto Oceanográfico da Universidade de São Paulo (IOUSP) em apoio às atividades da componente oceânica do Instituto Nacional de Ciência e Tecnologia para mudanças climáticas (INCT-Clima), tem-se o interesse de manter uma boia fundeada para monitoramento meteoceanográfico da Bacia de Santos. O equipamento consiste basicamente em uma boia meteoceanográfica similar àquela utilizada no Projeto PIRATA, sendo a boia ATLAS a qual possui um grande sucesso de atuação no cenário internacional (BOURLÈS et al., 2008).

A estrutura da boia do modelo ATLAS-B possui forma toroidal com aproximadamente 2,3m de diâmetro e foi confeccionada utilizando fibra de vidro e reforços internos de aço. A estrutura da boia do modelo MINITRIAXIS também foi confeccionada utilizando fibra de vidro, porém esta possui forma esférico-discoide e diâmetro de aproximadamente $1,2 \mathrm{~m}$.

Em ambas as boias, existem aparatos que permitem a instalação de equipamentos auxiliares para medição de outros parâmetros ambientais que não são medidos pela UMM, tais como Acoustic Doppler Current Profiler (ADCP - Perfilador de Corrente Doppler Acústico), estação meteorológica, sensores de medição de temperatura e salinidade, entre outros. A UMM é composta por um sensor de medição de movimento (pitch, roll e aceleração vertical); uma placa para aquisição e processamento dos dados do sensor de movimento, armazenamento e gerenciamento dos dados oriundos de outros sensores acoplados, um Global Positioning System (GPS - Sistema de Posicionamento Global) e uma fonte reguladora de tensão. Contudo, os componentes eletrônicos utilizados não são de origem nacional, e para redução dos custos da produção da UMM foi elaborada uma pesquisa de mercado que possibilitou a aquisição de componentes que atendessem aos objetivos específicos da construção do equipamento em questão com elevada qualidade, eficiência e a um custo acessível.

\subsection{ESTUdOS NA ÁREA DE OCEANOGRAFIA}

Um dos principais objetivos da oceanografia é conhecer a circulação oceânica. As medições por si só não fornecem todas as informações requeridas, pois elas depreendem um conjunto muito grande de fatores que influenciam as correntes, sendo primordial para analise o isolamento de cada um desses fatores em sua análise da circulação. Em consequência, as medidas diretas de correntes em diversas profundidades no mar são normalmente acompanhadas de outras medições, de natureza oceanográfica e meteorológica. Na ciência oceanográfica, os navios oceanográficos, plataformas, barcos e boias são os tradicionais suportes dos instrumentos e fonte primordial de informações para a construção de qualquer estudo. O Quadro 1 apresenta alguns trabalhos elaborados nessa área utilizando métodos quantitativos.

Quadro 1: Métodos quantitativos na área de climatologia oceanográfica

\begin{tabular}{|c|c|c|}
\hline Tema & Técnicas Utilizadas & Autor(es) / Ano \\
\hline $\begin{array}{c}\text { Comparação de Séries } \\
\text { Salinidade da Água }\end{array}$ & Séries Temporais & Echeverry \& Toloi (2000) \\
\hline $\begin{array}{c}\text { Medições de Agitação } \\
\text { Marítima }\end{array}$ & $\begin{array}{c}\text { Índice de Concordância, Viés } \\
\text { e Erro Quadrático Médio }\end{array}$ & Capitão \& Fortes (2001) \\
\hline $\begin{array}{c}\text { Estimativa da Temperatura } \\
\text { do Ar }\end{array}$ & Séries Temporais & $\begin{array}{c}\text { Cavalcanti, Silva \& Souza } \\
\text { (2006) }\end{array}$ \\
\hline $\begin{array}{c}\text { Meso-escala da Corrente da } \\
\text { Ressurgência }\end{array}$ & $\begin{array}{c}\text { Modelo Regional Orientado } \\
\text { por Feições }\end{array}$ & Calado (2006) \\
\hline Climatologia da & Análise Exploratória de & Da Silva, Dourado e \\
\hline
\end{tabular}




\begin{tabular}{|c|c|c|}
\hline Ressurgência & Dados & Candella (2006) \\
\hline $\begin{array}{c}\text { Temperatura da Superfície } \\
\text { do Oceano }\end{array}$ & $\begin{array}{c}\text { Análise Exploratória de } \\
\text { Dados }\end{array}$ & Cordeiro (2009) \\
\hline $\begin{array}{c}\text { Perda do Sinal Acústico na } \\
\text { Presença da Ressurgência }\end{array}$ & $\begin{array}{c}\text { Modelo Numérico } \\
\text { Hidrodinâmico }\end{array}$ & Teixeira (2011) \\
\hline $\begin{array}{c}\text { Temperatura de Superfície } \\
\text { do Mar }\end{array}$ & Séries Temporais & Laurindo \& Polito (2015) \\
\hline $\begin{array}{c}\text { Cálculo do Perfil Sintético da } \\
\text { Temperatura dos Oceanos }\end{array}$ & Série de Modos Normais & Mendonça et al. (2018) \\
\hline $\begin{array}{c}\text { Variabilidade Superficial de } \\
\text { Temperatura e Altimetria }\end{array}$ & Modelo Regional Oceânico \\
\hline
\end{tabular}

\section{METODOLOGIA}

Uma série temporal é um conjunto de observações sobre uma variável obtidas sequencialmente ao longo do tempo e registradas em intervalos regulares. Deste modo, podese dizer que uma série temporal é a produção particular de um processo estocástico (BOX et al., 2015).

Para elaborar uma previsão temporal, é interessante que alguns passos devam ser tomados. Primeiramente, deve-se optar por avaliar a série graficamente, podendo ser levantados alguns pressupostos. Em seguida, aplicar testes para verificação de estacionariedade, possível sazonalidade e, logo após, realizar o ajuste dos modelos (MORETTIN \& TOLOI, 2006). Para avaliação dos métodos, tem-se as métricas de previsão.

\subsection{COEFICIENTE DE CORRELAÇÃO DE SPEARMAN}

O coeficiente de Spearman é um teste não paramétrico baseado na correlação de postos (CORDER \& FOREMAN, 2014). Os dados são analisados em pares que são postos ou que podem ser convertidos em postos, deste modo, mede a intensidade da relação entre variáveis ordinais (usa, em vez do valor observado, apenas a ordem das observações). Este teste pode ser usado para detectar algumas relações que não são lineares. Esta medida de correlação é dada por:

$$
\rho=1-\frac{6 \sum d^{2}}{n\left(n^{2}-1\right)}
$$

Se os postos da variável x são exatamente iguais aos postos da variável y, então todos as diferenças (d) serão zero, e $\rho$ será igual a 1. Se coeficiente $\rho$ de Spearman for menor que zero, a série é estacionaria.

\subsection{KRUSKal-WaLlis}

Sazonalidade são oscilações de acréscimos ou decréscimos que se repetem em determinados períodos de tempo, que podem coincidir com o calendário anual, mensal, semanal ou períodos específicos definidos. Resultam primariamente por naturalidade, mas também podem ser causadas pelo comportamento humano. Um estudo de sazonalidade tem por objetivo analisar o comportamento típico de uma série temporal. Para tanto, esta análise deve ser realizada em intervalos de tempos regulares.

O processo de realização deste teste tem início com a ordenação do conjunto de valores da variável, onde cada observação recebe um posto correspondente. Em seguida, somam-se os postos de cada grupo (no caso meses) e calcula-se a estatística H. Esta estatística pode ser aproximada por uma distribuição de probabilidade Qui-Quadrado, com k 
- 1 graus de liberdades, onde k é o número de meses, nj é o número de casos em cada mês j e $\mathrm{N}=\sum$ nj (SIEGEL \& CASTELLAN, 1988).

$$
\mathrm{H}=\frac{12}{\mathrm{n}(\mathrm{n}+1)} * \sum_{\mathrm{i}=1}^{\mathrm{k}} \frac{\mathrm{R}_{\mathrm{j}}^{2}}{\mathrm{n}_{\mathrm{j}}}-3(\mathrm{n}+1
$$

A hipótese nula de não existência de sazonalidade é rejeitada se a estatística $H$ for maior ou igual ao valor crítico retirado dado pela distribuição Qui-Quadrado.

\subsection{Modelos de Alisamento}

Segundo Morettin e Toloi (1981), uma grande classe de métodos de previsão, que tentam tratar as causas de flutuações em séries de tempo, é a dos Métodos de Alisamento (também conhecidos como Métodos de Suavização). Essas técnicas são usadas para se obter o padrão básico da série assumindo que os valores extremos são representados pela aleatoriedade (DOUKHAN, 2018). Deste modo, os modelos utilizados neste trabalho foram: Alisamento Exponencial Simples, Alisamento Exponencial Linear de Brown, Alisamento Exponencial de Holt e Alisamento Exponencial Sazonal de Holt-Winters. Todas as previsões foram feitas um passo à frente.

\subsubsection{Alisamento Exponencial Simples (AES)}

Os modelos AES são apropriados para séries que são caracterizadas localmente pelo seu nível e também estacionárias. O método consiste em ponderar todos os valores históricos da série com pesos sucessivamente menores à medida que estes se afastam do valor mais recente, eliminando assim uma deficiência do método das médias móveis (BROCKWELL \& RICHARD, 2016).

$\overline{\mathrm{Z}_{\mathrm{t}}}$ é o valor alisado no estágio t e a previsão dos h valores futuros é dada pelo último valor exponencialmente suavizado. Dessa forma:

$$
\begin{gathered}
\overline{\mathrm{Z}}_{\mathrm{t}}=\alpha \mathrm{Z}_{\mathrm{t}}+(1-\alpha) \mathrm{Z}_{\mathrm{t}-1} \\
\widehat{\mathrm{Z}}(\mathrm{h})=\overline{\mathrm{Z}}_{\mathrm{t}}
\end{gathered}
$$

\subsubsection{Alisamento Exponencial Linear de Brown (AELB)}

Não é apropriado a utilização do AES quando uma série apresenta tendência linear, pois este modelo gera previsões que subestimam, ou superestimam, os valores reais. O AELB é um método semelhante ao AES, porém, indicado para séries que apresentam tendência. É usada uma única constante de alisamento no processo de atualização do nível e da tendência, representados respectivamente pelos parâmetros $\widehat{b}_{1, t}$ e $\widehat{b}_{2, t}$. Consiste em calcular um segundo valor exponencialmente alisado, dado pela equação (BROCKWELL \& RICHARD, 2016):

$$
\overline{\bar{Z}}_{t}=\alpha \overline{\mathrm{Z}}_{\mathrm{t}}+(1-\alpha) \overline{\overline{\mathrm{Z}}}_{\mathrm{t}-1}
$$

E os parâmetros das estimativas de nível e tendência são dados por:

$$
\begin{gathered}
\hat{\mathrm{b}}_{1, \mathrm{t}}=2 \overline{\mathrm{Z}}_{\mathrm{t}}-\overline{\overline{\mathrm{Z}}}_{\mathrm{t}} \\
\hat{\mathrm{b}}_{2, \mathrm{t}}=\frac{\alpha}{1-\alpha}\left(\overline{\mathrm{Z}}_{\mathrm{t}}-\overline{\overline{\mathrm{Z}}}_{\mathrm{t}-1}\right)
\end{gathered}
$$

Deste modo, a previsão final dos h valores futuros é dada por: 


$$
\widehat{\mathrm{z}}_{\mathrm{t}}(\mathrm{h})=\widehat{\mathrm{b}}_{1, \mathrm{t}}+\widehat{\mathrm{b}}_{2, \mathrm{t}}
$$

\subsubsection{Alisamento Exponencial de Holt (AEH)}

As características do AEH são similares a do AES, porém, além de suavizar o nível, uma nova constante é utilizada para a modelagem da tendência da série, resultando em dois coeficientes de alisamento $\alpha$ e $\beta$ que representam, respectivamente, o nível e a tendência. A equação do modelo é representada por (BROCKWELL \& RICHARD, 2016):

$$
\begin{aligned}
& \overline{\mathrm{Z}}_{\mathrm{t}}=\alpha \mathrm{Z}_{\mathrm{t}}+(1-\alpha)\left(\overline{\mathrm{Z}}_{\mathrm{t}-1}+\widehat{\mathrm{T}}_{\mathrm{t}-1}\right) \\
& \widehat{\mathrm{T}}_{\mathrm{t}}=\beta\left(\overline{\mathrm{Z}}_{\mathrm{t}}+\overline{\mathrm{Z}}_{\mathrm{t}-1}\right)+(1-\beta) \widehat{\mathrm{T}}_{\mathrm{t}-1}
\end{aligned}
$$

E a previsão final é dada por:

$$
\widehat{\mathrm{Z}}(\mathrm{h})=\overline{\mathrm{Z}}_{\mathrm{t}}+\mathrm{h} \widehat{\mathrm{T}}_{\mathrm{t}}
$$

\subsubsection{Alisamento Exponencial Sazonal de Holt-Winters (AESHW)}

É utilizada em séries temporais que apresentam padrões de comportamento mais complexos. Este modelo apresenta coeficientes para estimar o nível, a tendência e a sazonalidade, representados respectivamente por $\alpha, \beta$ e $\gamma$. A formulação do modelo sazonal aditivo é dada por (BROCKWELL \& RICHARD, 2016):

$$
\begin{gathered}
\widehat{\mathrm{S}}_{\mathrm{t}}=\gamma\left(\mathrm{Z}_{\mathrm{t}}-\overline{\mathrm{Z}}_{\mathrm{t}}\right)+(1-\gamma) \widehat{\mathrm{S}}_{\mathrm{t}-s} \\
\overline{\mathrm{Z}}_{\mathrm{t}}=\alpha\left(\mathrm{Z}_{\mathrm{t}}-\widehat{\mathrm{S}}_{\mathrm{t}-s}\right)+(1-\alpha)\left(\overline{\mathrm{Z}}_{\mathrm{t}-1}+\widehat{\mathrm{T}}_{\mathrm{t}-1}\right) \\
\widehat{\mathrm{T}}_{\mathrm{t}}=\beta\left(\overline{\mathrm{Z}}_{\mathrm{t}}+\overline{\mathrm{Z}}_{\mathrm{t}-1}\right)+(1-\beta) \widehat{\mathrm{T}}_{\mathrm{t}-1}
\end{gathered}
$$

E a previsão final é dada pela soma dos três parâmetros calculados:

$$
\widehat{\mathrm{Z}}(\mathrm{h})=\overline{\mathrm{Z}}_{\mathrm{t}}+\mathrm{h} \widehat{\mathrm{T}}_{\mathrm{t}}+\widehat{\mathrm{S}}_{\mathrm{t}+\mathrm{h}-\mathrm{s}}
$$

\subsection{MÉtricas de Previsão}

Quando previsões periódicas são efetuadas, é importante monitorar os erros para determinar se estão dentro de limites razoáveis. Existem algumas métricas de previsão para avaliar a qualidade dos modelos propostos, das quais podem envolver valores absolutos ou percentuais.

\subsubsection{Erro Quadrático Médio (EQM)}

O EQM é muito útil na comparação de estimadores (MENDONÇA et al., 2012). Esta métrica é determinada somando os erros previstos ao quadrado e dividindo pelo número de previsões. Pode ser expresso pela seguinte equação (DE OLIVEIRA et al., 2010):

$$
E Q M=\frac{\sum\left(y_{i}-\hat{y}_{i}\right)^{2}}{n}
$$

\subsubsection{Média Absoluta Percentual de Erro (MAPE)}

Se a previsão do modelo é medida em milhares, os valores do EQM serão muito 
grandes. A utilização do MAPE é uma alternativa eficaz para resolução deste problema. Esta medida é a média da diferença absoluta entre os valores previstos e atuais, expressa em percentagem dos valores atuais. Assim, se existem previsões e valores reais para n períodos, o erro médio percentual absoluto é (DE OLIVEIRA et al., 2010):

$$
\text { EMPA }=\frac{\sum\left|\frac{y_{i}-\hat{y}_{i}}{y_{i}}\right| * 100}{n}
$$

\section{RESULTADOS}

Com o intuito de observar as características das variações das séries temporais, como por exemplo, sazonalidade, estacionariedade, variabilidade e pontos atípicos, é de extrema importância a construção de um gráfico. O comportamento das séries, ao longo do período observado, será analisado inicialmente através da análise descritiva dos dados. Notase que as séries não apresentam tendência de crescimento ou decrescimento, indicando serem séries estacionárias, conforme o Gráfico 1 abaixo.

Gráfico 1: Valores Observados para Temperatura e Salinidade Mensal.

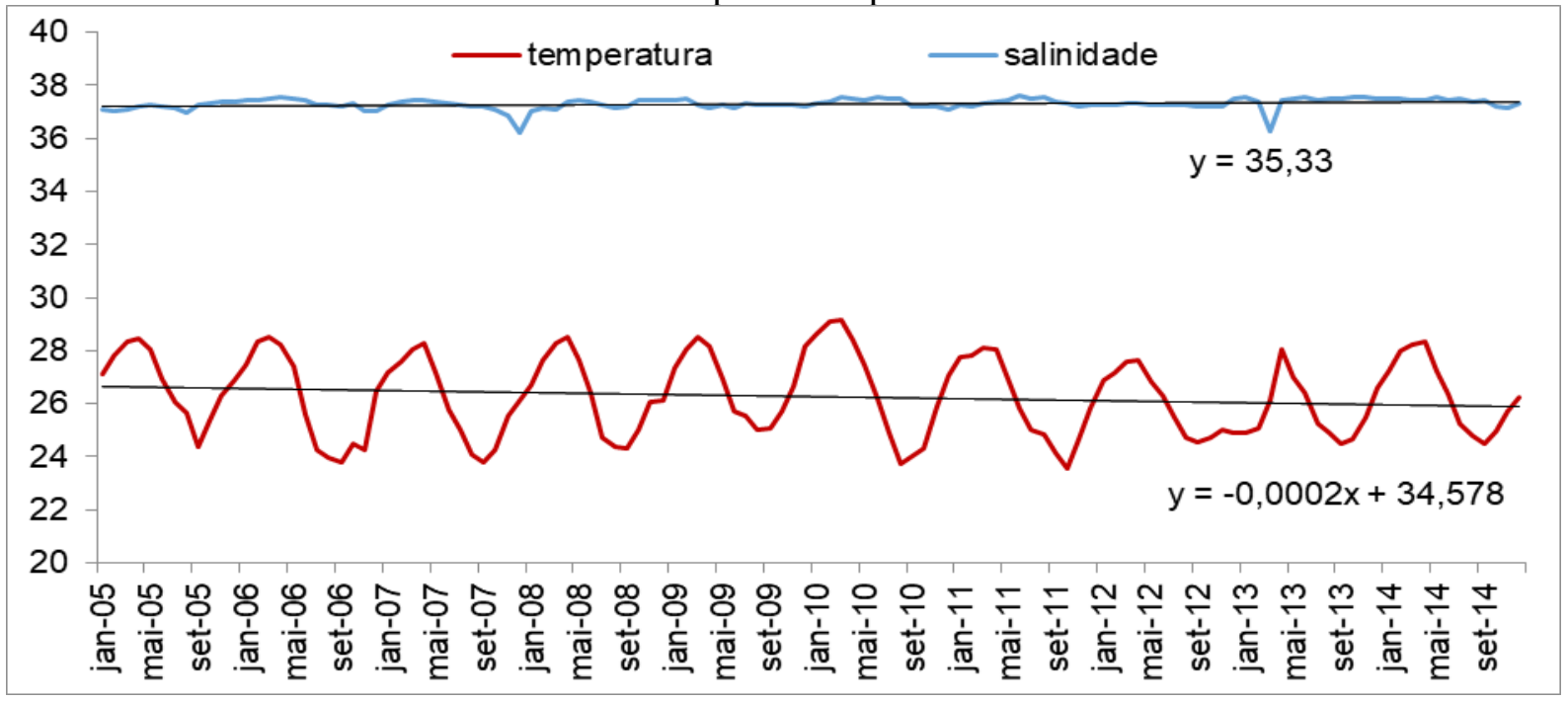

Fonte: Programa Global Ocean Observing System, 2015.

Pode-se também observar as retas de regressão que compõem o Gráfico 1, não possuem indicação de crescimento ou decrescimento, visto que seus coeficientes angulares são aproximadamente nulos.

Realizou-se uma análise de componente de tendência para verificar se as variáveis temperatura e salinidade apontam, de forma precisa, a não existência de tendência nas séries em estudo. Nesta análise, utilizou-se o coeficiente de correlação de Spearman, que para valores negativos indica que não existe tendência, confirmando-se que as séries temporais em estudo não apresentam tendência forte de crescimento ou decrescimento. O Quadro 1 apresenta os resultados.

Quadro 2: Coeficiente de Correlação de Spearman.

\begin{tabular}{|c|c|c|}
\hline Variável & Valor de $\rho$ & Conclusão \\
\hline Temperatura & $-0,142$ & Estacionária \\
\hline Salinidade & $-0,017$ & Estacionária \\
\hline
\end{tabular}


Observou-se que a salinidade nessa área do oceano atlântico não possui uma variação cíclica que é característica das séries que possuem sazonalidade. Tendo observado que tanto visualmente, quanto que através do teste de Kruskal-Wallis, não foi captada nenhuma componente sazonal (Quadro 2).

Já na estatística teste para a temperatura média mensal, é rejeitada a hipótese nula de que, em média, os doze meses sejam iguais. Dessa forma, fica evidente que, pelo menos um mês possui uma temperatura média diferente das demais. Os resultados compilados para a temperatura e salinidade estão descritos a seguir no Quadro 2.

Quadro 3: Teste de Kruskal-Wallis.

\begin{tabular}{|c|c|c|c|c|c|c|}
\hline Variável & $\begin{array}{c}\text { Valor } \\
\text { Calculado }\end{array}$ & $\begin{array}{c}\text { Valor } \\
\text { Tabelado }\end{array}$ & p-valor & $\begin{array}{c}\text { Graus de } \\
\text { Liberdade }\end{array}$ & $\begin{array}{c}\text { Nível de } \\
\text { Significânci } \\
\text { a }\end{array}$ & Conclusão \\
\hline $\begin{array}{c}\text { Temperatur } \\
\text { a }\end{array}$ & 97,246 & 19,675 & $6,3 \times 10^{-16}$ & 11 & 0,05 & Sazonal \\
\hline Salinidade & 11,691 & 19,675 & 0,387 & 11 & 0,05 & $\begin{array}{c}\text { Não } \\
\text { Sazonal }\end{array}$ \\
\hline
\end{tabular}

As séries de temperatura e salinidade médias mensais foram divididas em duas partes. O período entre janeiro de 2005 e dezembro de 2012 foi utilizado para a estimação dos parâmetros dos modelos, enquanto que, o período entre janeiro de 2013 e dezembro de 2014 foi utilizado para a verificação, ou seja, para a escolha do melhor modelo através da comparação dos menores valores do EQM e do MAPE para a previsão da série. Para estabelecer os parâmetros (ou as constantes de alisamento) foi utilizado o Software R (JONATHAN \& KUNK-SIK, 2008) com o objetivo de aperfeiçoar a escolha dos mesmos, revelados pelo Quadro 3.

Quadro 4: Valores das Constantes de Alisamento.

\begin{tabular}{|c|c|c|c|c|c|c|}
\hline \multirow{2}{*}{ Modelos } & \multicolumn{7}{|c|}{ Constantes } \\
\cline { 2 - 7 } & \multicolumn{3}{|c|}{ Temperatura } & \multicolumn{3}{c|}{ Salinidade } \\
\cline { 2 - 7 } & $\alpha$ & $\beta$ & $\gamma$ & $\alpha$ & $\beta$ & $\gamma$ \\
\hline AES (EQM) & 0,99 & - & - & 0,82 & - & - \\
\hline AES (MAPE) & 0,99 & - & - & 0,99 & - & - \\
\hline AELB (EQM) & 0,95 & - & - & 0,43 & - & - \\
\hline AELB (MAPE) & 0,99 & - & - & 0,99 & - & - \\
\hline AEH (EQM) & 0,90 & 0,90 & - & 0,80 & 0,10 & - \\
\hline AEH (MAPE) & 0,90 & 0,90 & - & 0,90 & 0,10 & - \\
\hline AESHW & 0,78 & 0,0007 & 0,98 & 0,70 & 0,00 & 0,62 \\
\hline
\end{tabular}

Os Gráficos 2 e 3 apresentam a comparação entre os valores observados e as previsões obtidas pelos respectivos parâmetros EQM e MAPE em cada modelo aplicado para variável temperatura dentro do período compreendido entre janeiro de 2013 e dezembro de 2014.

Gráfico 2: Previsão da Temperatura pelos Parâmetros Avaliados no EQM. 


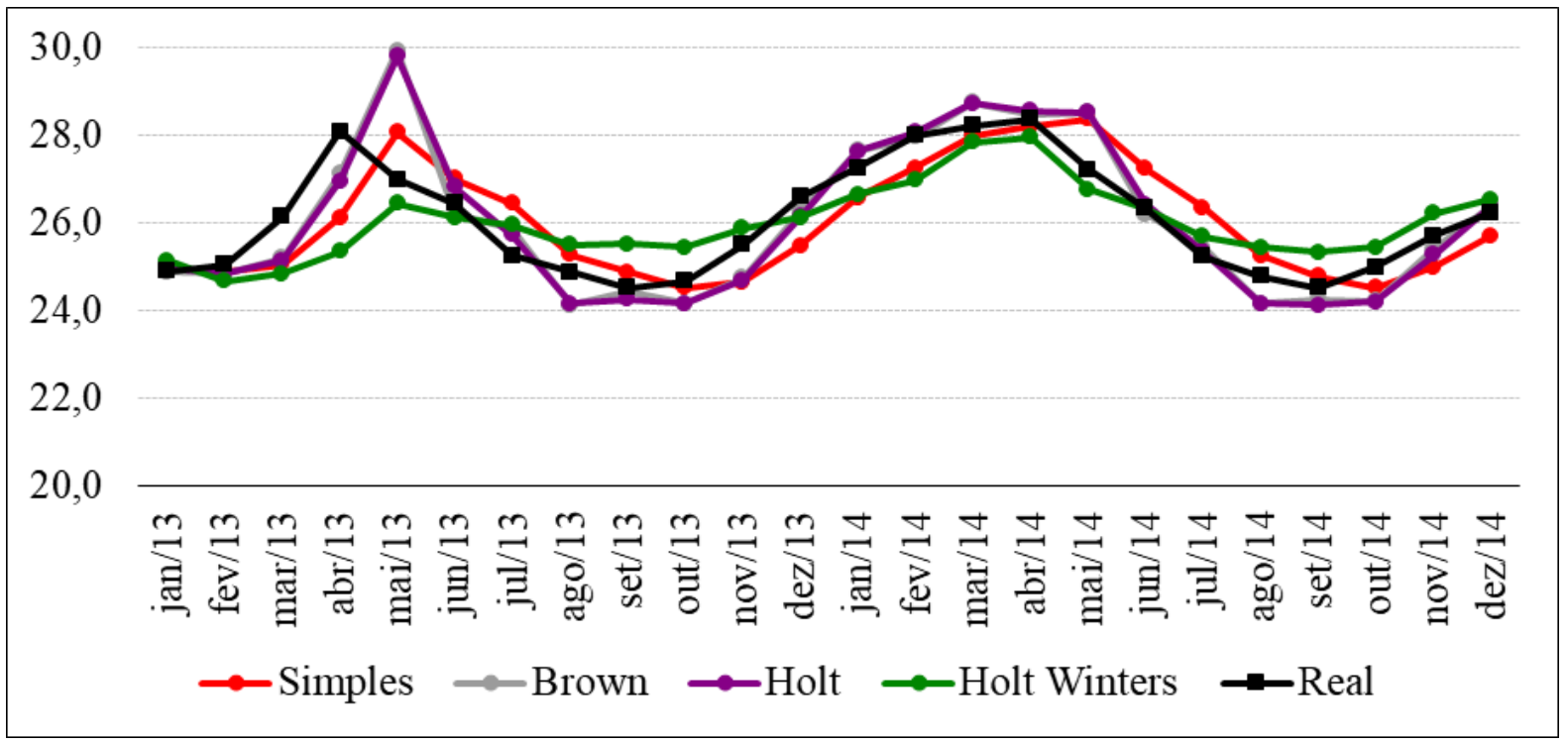

Gráfico 3: Previsão da Temperatura pelos Parâmetros Avaliados no MAPE.

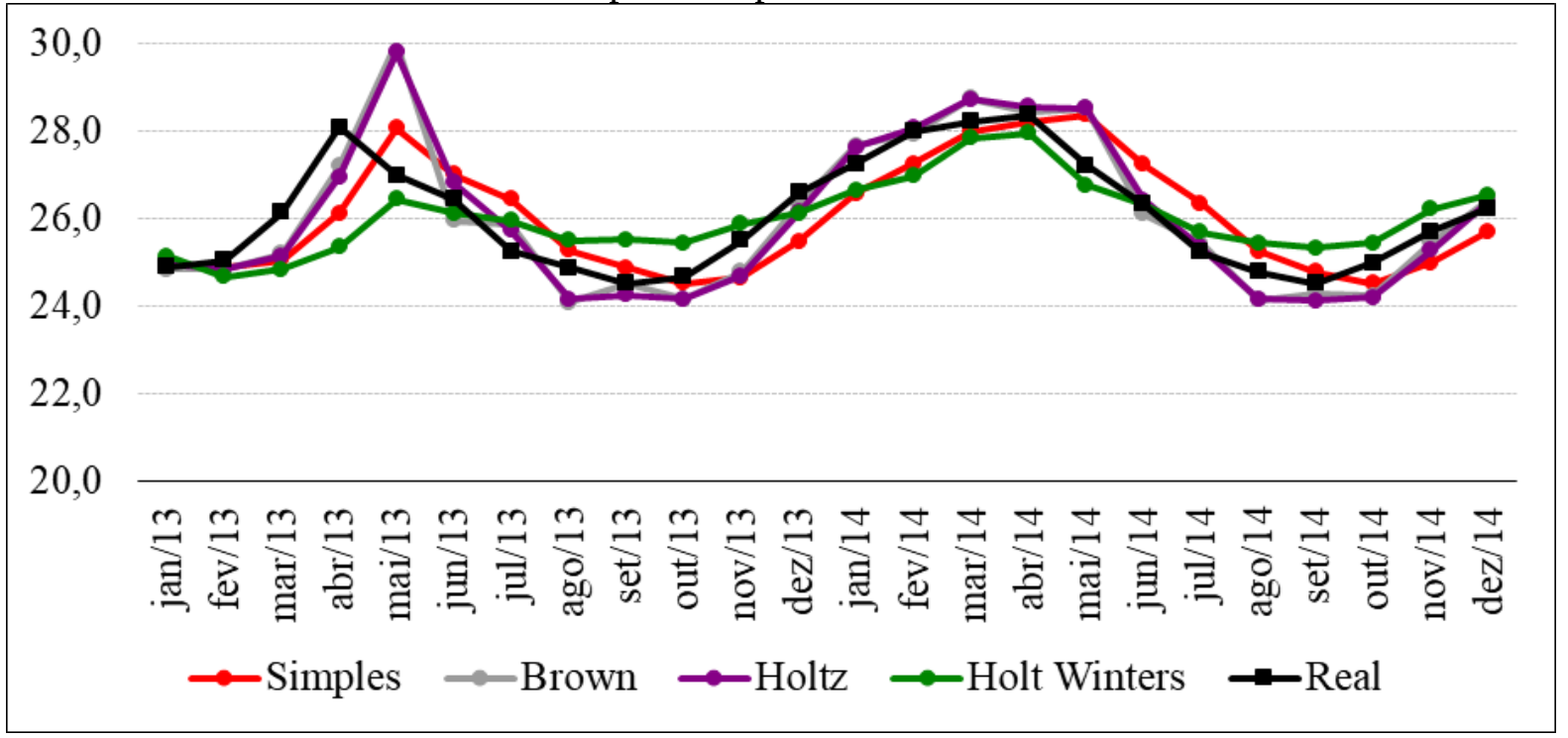

Os Gráficos 4 e 5 apresentam a comparação entre os valores observados e as previsões obtidas pelos respectivos parâmetros EQM e MAPE em cada modelo aplicado para variável salinidade dentro do período compreendido entre janeiro de 2013 e dezembro de 2014.

Gráfico 4: Previsão da Salinidade pelos Parâmetros Avaliados no EQM. 


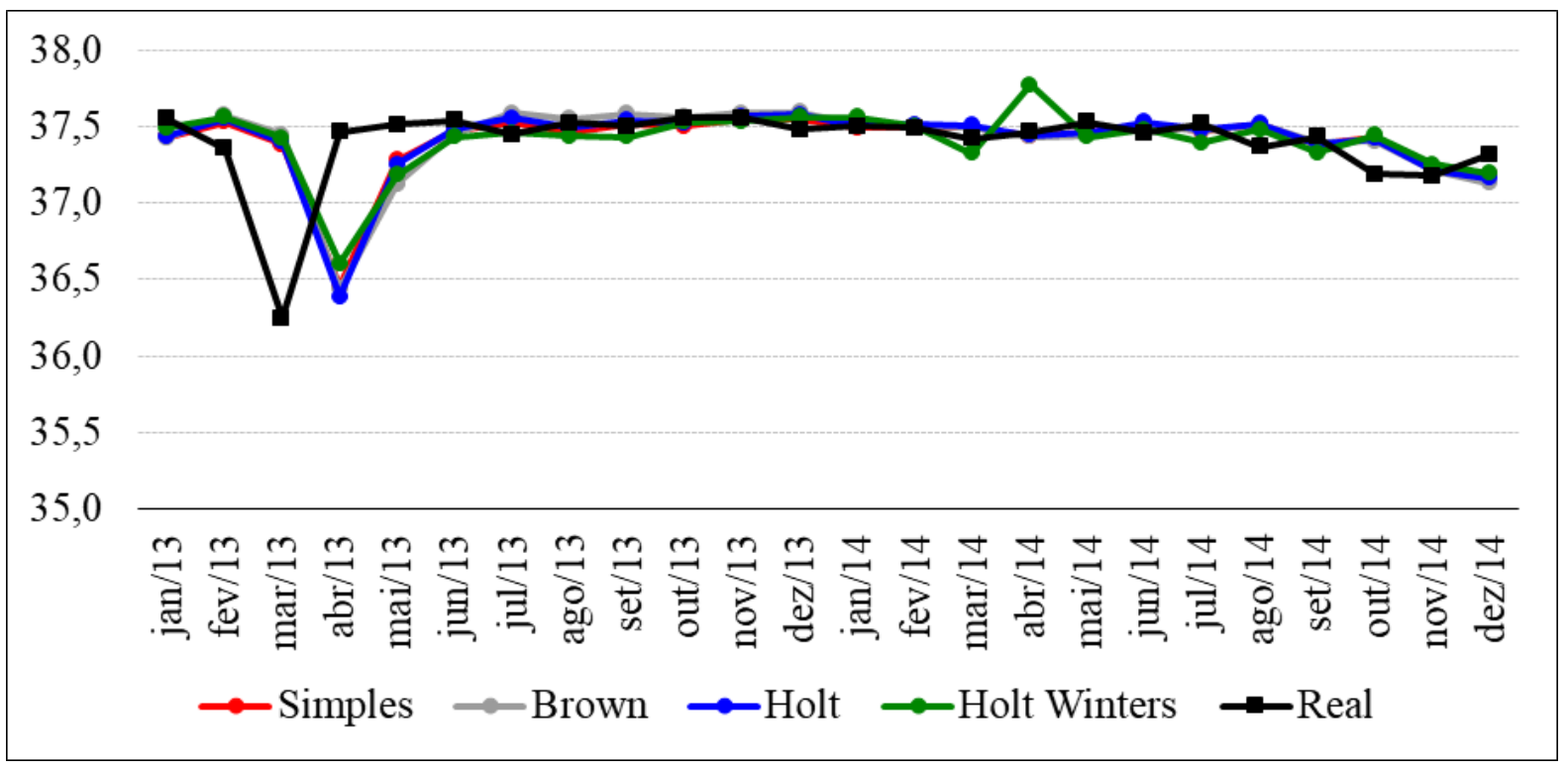

Gráfico 5: Previsão da Salinidade pelos Parâmetros Avaliados no MAPE.

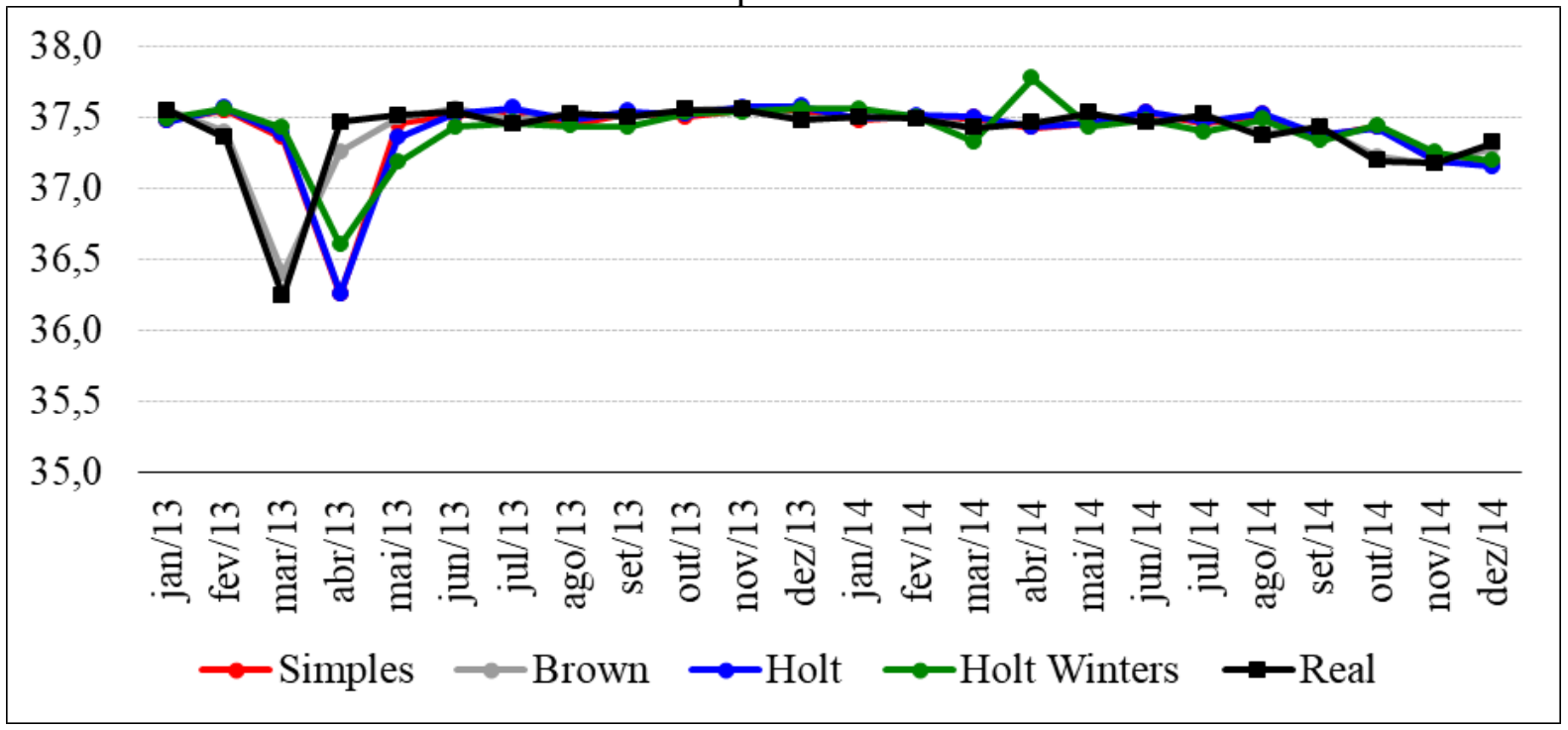

Na aplicação dos dados aos Métodos de Alisamento Exponencial, por intermédio do EQM e do MAPE, identificou-se que o modelo de AELB é o mais indicado para representar o comportamento da série temporal das temperaturas médias mensais, por esta apresentar forte componente sazonal e não apresentar tendência, e o modelo AES o mais indicado para a série temporal das salinidades médias mensais, pois este não considera o componente sazonal e também não apresenta tendência. Tais resultados podem ser observados através do Quadro 4 a seguir:

Quadro 5: Valores das Métricas de Previsão

\begin{tabular}{|c|c|c|c|c|}
\hline \multirow{2}{*}{ Modelos } & \multicolumn{2}{|c|}{ Temperatura } & \multicolumn{2}{c|}{ Salinidade } \\
\cline { 2 - 5 } & EQM & MAPE & EQM & MAPE \\
\hline AES & 0,6599 & 2,5758 & 0,1069 & 0,4442 \\
\hline AELB & 0,6577 & 2,1181 & 0,1207 & 0,5415 \\
\hline AEH & 0,6580 & 2,1988 & 0,1150 & 0,4617 \\
\hline AESHW & 0,6802 & 4,2161 & 0,1075 & 4,2161 \\
\hline
\end{tabular}


De acordo com LEWIS (1997), o potencial de previsão do MAPE pode ser relacionado da seguinte forma: menor que $10 \%$ são previsões potencialmente muito boas; entre $10 \%$ e $20 \%$ são previsões potencialmente boas; entre $20 \%$ e $30 \%$ são previsões potencialmente razoáveis; e maior que $30 \%$ são previsões potencialmente imprecisas. Sendo assim, conclui-se que todos os modelos obtiveram previsões potencialmente muito boas neste estudo.

\section{CONSIDERAÇÕES FINAIS}

Objetivou-se neste trabalho a utilização de métodos estatísticos de análise de séries temporais para a previsão das temperaturas médias mensais e para a previsão das salinidades médias mensais na posição geográfica $19^{\circ} \mathrm{S} 34^{\circ} \mathrm{W}$.

O método de Alisamento Exponencial é uma técnica de previsão, que considera o princípio da estimação recursiva, e não exige qualquer transformação nos dados originais para que, por exemplo, respeitem uma distribuição normal ou sejam estacionários. A aplicação deste método concentra-se apenas no uso de fórmulas com as quais as previsões são obtidas através de alisamento dos valores passados. Assim sendo, as principais características deste método estão relacionadas a facilidade de uso e a aplicabilidade a um grande número de séries temporais. A utilização desse tipo de metodologia pode render resultados muito satisfatórios dependendo de condições como tendência, sazonalidade, ciclo e componente aleatório, além de permitir uma boa compreensão dos resultados.

Em geral, para a previsão de variáveis meteorológicas, utilizam-se modelos numéricos da circulação geral da atmosfera e oceano. Estes modelos necessitam de uma grande quantidade de informações e capacidade de processamento computacional. Como resultados são produzidas previsões de temperatura, vento, umidade e outros, em uma escala de tempo mais curta.

Pode-se considerar que nenhum modelo obteve fraco desempenho (LEWIS, 1997). Para a temperatura, o modelo que obteve melhor ajuste foi o Alisamento Exponencial Linear de Brown, e para salinidade, o modelo de Alisamento Exponencial Simples foi o que apresentou melhor resultado.

Uma outra hipótese para realização deste trabalho, ainda no âmbito da previsão de séries temporais seria a utilização dos modelos Box-Jenkis e as técnicas de machine learning (JAMES, et al., 2013), como as Máquinas de Vetores de Suporte (SUYKENS \& VANDEWALLE, 1999).

Também seria interessante testar outras boias com diferentes localizações geográficas, visto que a costa brasileira é muito ampla e as tendências climáticas tem variado bastante ao longo das últimas décadas.

\section{REFERÊNCIAS BIBLIOGRÁFICAS}

[1] Banco de Dados Oceanográficos. Dados oceanográficos de temperatura, salinidade e superfície. Disponível em: http://www.goosbrasil.org/pirata/dados. Acessado em: 10 set. 2014.

[2] BOURLÈS, B. et al. The PIRATA program: History, accomplishments, and future directions. Bulletin of the American Meteorological Society, v. 89, n. 8, p. 1111-1126, 2008.

[3] BOX, G. E. P. et al. Time series analysis: forecasting and control. John Wiley \& Sons, 2015. 
[4] BROCKWELL, P.; RICHARD, D. Introduction to time series and forecasting. Springer, 2016.

[5] CALADO, L. Dinâmica da interação da atividade de meso-escala da Corrente do Brasil com o fenômeno da ressurgência costeira ao largo de Cabo Frio e Cabo de São Tomé, RJ. Tese de Doutorado. Universidade de São Paulo. 2006.

[6] CAPITÃO, R.; FORTES, C. Análise comparativa entre estimativas do modelo SWAN e medições de agitação marítima efectuadas na Praia da Amoreira, Portugal. Revista de Gestão Costeira Integrada-Journal of Integrated Coastal Zone Management, v. 11, n. 3, p. 283-296, 2011.

[7] CAVALCANTI, E. P.; SILVA, V. de P. R.; SOUSA, F. de A. S. Programa computacional para a estimativa da temperatura do ar para a região Nordeste do Brasil. Revista Brasileira de Engenharia Agrícola e Ambiental, v. 10, n. 1, p. 140-147, 2006.

[8] CODATO, G. A. S. et al. A influência da frente térmica da ressurgência costeira de cabo frio na perda do sinal acústico: um estudo numérico. $X$ Encontro de Tecnologia Acústica Submarina-ETAS, 2011.

[9] CORDEIRO, N. G. F. Estudo do afloramento ibérico: filamentos e climatologia superficial. Dissertação (Mestrado). Universidade de Aveiro. Departamento de Física. 2009.

[10] CORDER, G. W.; FOREMAN, D. I. Nonparametric statistics: A step-by-step approach. John Wiley \& Sons, 2014.

[11] DE ANDRADE, A. F.; COSTA, D. P. Pesquisa e desenvolvimento de boias meteoceanográficas. In: V Simpósio Brasileiro de Oceanografia, Santos. CD de Resumos, 2011.

[12] DE OLIVEIRA, A. C. S. et al. Aplicação de redes neurais artificiais na previsão da produção de álcool. Ciênc. Agrotec. [online], vol.34, n.2, pp.279-284. ISSN 14137054. 2010.

[13] DEFANT, A. Physical oceanography. Pergamon, 1961.

[14] DOUKHAN, P. Stochastic models for time series. Berlin: Springer, 2018.

[15] ECHEVERRY, G. E. S.; TOLOI, C. M. de C. Testes para comparação de séries temporais: uma aplicação a séries de temperatura e salinidade da água, medidas em profundidades diferentes. Rev. Bras. Estat, p. 51-80, 2000.

[16] Global Sea Level Observing System. Informações sobre sistemas de monitoramento oceanográfico. Disponível em: https://www.gloss-sealevel.org. Acesso em: 20 out. 2015.

[17] JAMES, G. et al. An introduction to statistical learning. New York: Springer, 2013.

[18] JONATHAN, D. C.; KUNG-SIK, C. Time series analysis with applications in R. SpringerLink, Springer eBooks, 2008.

[19] LAURINDO, L.; POLITO, P. Cálculo de perfis sintéticos de temperatura para os oceanos entre 39,5S e 39,5N a partir de dados de satélite, climatológicos e in situ. In: XIV Congresso Latino-Americano de Ciências do Mar, Balneário Camboriú, SC., 2011. 
[20] LEWIS, C. D. Demand Forecasting and Inventory Control. New York: Wiley, 157p 1997.

[21] EMÍLSSON, I. The shelf and coastal waters off southern Brazil. Boletim do Instituto Oceanográfico, v. 11, n. 2, p. 101-112, 1961.

[22] MARINHA DO BRASIL, Navio de Pesquisa Hidroceanográfico "Vital de Oliveira" realiza rodízio e manutenção de boias meteoceanográficas. Disponível em: https://www.marinha.mil.br/noticias/navio-de-pesquisa-hidroceanografico-vitalde-oliveira-realiza-rodizio-e-manutencao-de-boias. Acesso em: 14 mai. 2019.

[23] MASCARENHAS JR, A. da S.; MIRANDA, L. B. de; ROCK, N. J. A study of the oceanographic conditions in the region of Cabo Frio. Fertility of the Sea, v. 1, p. 285308, 1971.

[24] MENDONÇA, L. F. et al. Análise da Variabilidade Superficial de Temperatura e Altimetria no Oceano Atlântico Sudoeste durante o Ano de 2012. Revista Brasileira de Cartografia, v. 70, n. 3, p. 1158-1176, 2018.

[25] MORETTIN, P. A.; TOLOI, C. M. de C. Modelos para previsão de séries temporais. Instituto de Matemática Pura e Aplicada (IMPA), 1981.

[26] MORETTIN, P. A.; TOLOI, C. M. de C. Análise de séries temporais. In: Análise de séries temporais. ABE, Blucher, 2006.

[27] SIEGEL, S.; JOHN JR, N. C. Nonparametric Statistics for the Behavioral Sciences. New York, 6. McGraw-Hill, 1988.

[28] SUYKENS, J. A. K.; VANDEWALLE, J. Least squares support vector machine classifiers. Neural processing letters, v. 9, n. 3, p. 293-300, 1999.

[29] TEAM, R. Core et al. R: A language and environment for statistical computing. 2013.

[30] TEIXEIRA, P. M. Uso de Técnicas de Mudança de Periodicidade de Séries Temporais para Previsão da Temperatura de Superfície do Mar do Oceano Atlântico. Dissertação (Mestrado), Centro Federal de Educação Tecnológica Celso Suckow da Fonseca, CEFET/RJ, Rio de Janeiro. 2014. 